\title{
"Population structure of Drug-Susceptible, -Resistant and ESBL-producing Escherichia coli from Community-Acquired Urinary Tract Infections"
}

Frederik Boëtius Hertz ${ }^{1,2^{*}}$, Jesper Boye Nielsen ${ }^{1}$, Kristian Schønning ${ }^{1}$, Pia Littauer ${ }^{1}$, Jenny Dahl Knudsen ${ }^{1}$, Anders Løbner-Olesen ${ }^{2}$ and Niels Frimodt-Møller ${ }^{3}$

\begin{abstract}
Background: Escherichia coli is the most common cause of urinary tract infection (UTI). The pathogenic isolates are becoming increasingly resistant to antibiotics; with a worldwide dissemination of resistant sequence types (ST). We characterized three different uropathogenic E. coli populations, from non-hospitalized patients to describe the genetic kinship between resistant and susceptible isolates. We studied the populations by use of multi-locus sequence typing (MLST) and abbreviated-multi locus variable number of tandem repeat analysis (a-MLVA). Urine samples submitted for testing, by general practitioners, were identified at Dept. of Clinical Microbiology at Hvidovre Hospital, Denmark, from Oct. 2011 to July 2012. We included 94 fully susceptible, 94 resistant (non-ESBL) and 98 Extended Spectrum Beta-lactamases- (ESBL)-producing E. coli isolates.

Results: The ESBL population was dominated vastly by ST131 (51 \%), ST38 (9 \%) and ST69 (6 \%). In the resistant group ST69 (18\%), ST73 (11\%) and ST131 (15\%) were the largest clusters. In the susceptible population more STs and a-MLVA codes were identified compared to the other groups and ST73 and ST95 were found as the only clusters with $16 \%$ and $6 \%$, respectively. Ninety-eight per cent of the ESBL-producing E. coli isolates were CTX-M-producers.

Conclusion: ST131 dominated the population of community-associated uropathogenic ESBL-producing E. coli, but was less frequent among non-ESBL-producing E. coli. The fully susceptible E. coli population was a much more diverse group than the resistant and ESBL-producing E. coli populations. Overall, these findings suggest that dominant ESBL-producing lineages are derived from UPEC lineages already established in the general UPEC population.
\end{abstract}

Keywords: Escherichia coli, Lineages, Urinary tract infection, Community-acquired, ESBL

\section{Background}

Escherichia coli is the most common Gram-negative extraintestinal pathogen and the primary cause of urinary tract infection (UTI) [1, 2]. It is a highly heterogonous species with certain lineages becoming increasingly resistant to antibiotics [2-5]. Thus, resistant $E$. coli isolates are emerging worldwide and especially $E$. coli producing

\footnotetext{
* Correspondence: fbh@dadlnet.dk

${ }^{1}$ Department of Clinical Microbiology, Hvidovre University Hospital, Copenhagen, Denmark

${ }^{2}$ Department of Biology, University of Copenhagen, Copenhagen, Denmark Full list of author information is available at the end of the article
}

extended-spectrum beta-lactamases (ESBLs) have been reported numerous times around the world $[2,6]$. Now E. coli isolates primarily produce ESBLs belonging to the enzyme family CTX-M $[2,6]$. The dissemination of ESBL-producing $E$. coli in hospital settings as well as in the community has been reported as spread of extraintestinal pathogenic E. coli (ExPEC) belonging to a limited number of lineages or sequence types (STs) $[2,6]$. However, only a few studies have specifically studied the heterogeneity among susceptible, resistant and ESBLproducing E. coli $[2-4,6]$. Interestingly, dominating ExPEC 
lineages, like ST73 and ST95 continue to be common causes of UTI, but are rarely multidrug resistant and seldom associated with ESBL production. These lineages have previously been sub-typed by different typing methods showing the existence of several subclones. An abbreviated-multi locus variable number of tandem repeat analysis (a-MLVA) method has previously been evaluated and proved efficient in the characterization of local ESBLproducing E. coli in hospital settings where the method typed congruent with multi-locus sequence typing (MLST) [7]. However, MLST, in spite of its clear advantage of having an internationally nomenclature, is laborious and needs sequencing where MLVA can be used as a fast and low cost method for screening larger population.

Therefore, we here present a descriptive investigation of three E. coli populations all from non-hospitalized patients. The characterization is performed by use of a-MLVA and subsequent MLST of identified a-MLVA codes.

\section{Methods}

\section{Research ethical approvals}

Approvals for this study were granted by the Danish Health and Medicines Authority, Statistics Denmark (DST) and The Regional Committee of Danish Data Protection Agency. Patient notification was declared not necessary by the Regional Committee of Health Research Ethics Committee (HVH-2012-017, J.nr. 3-3013-230/1/ KWH and H-4-2012-088).

\section{Strain collections}

The Department of Clinical Microbiology at Hvidovre Hospital, Denmark (DCM) provides services to more than 450 general practitioners in an area of approximately 950.000 inhabitants. From the $1^{\text {st }}$ of October 2011 to $30^{\text {th }}$ of June 2012 we collected 286 E. coli isolates from patient urine samples, submitted from general practices. Hence, from non-hospitalized patients, one unique strain was collected per patient and isolates were divided into three susceptibility groups: (i) ESBL-producing E. coli, (ii) E. coli resistant to at least one of 17 tested antibiotic (ampicillin, cefuroxime, aztreonam, ampicillin/clavulanic acid, piperacillin/tazobactam, mecillinam, ceftazidime, cefpodoxime, meropenem, ciprofloxacin, sulfamethoxazole, trimethoprim, tetracycline, gentamicin, tobramycin, nitrofurantoin, fosfomycin) but without an ESBL phenotype and (iii) fully susceptible E. coli. Thus, in this study we included 98 ESBL-producing E. coli isolates, 94 resistant isolates (non-ESBL) and 94 fully susceptible E. coli.

\section{Strain identification and susceptibility testing}

The UPEC isolates were all identified at species level by MALDI-TOF MS (Bruker, Germany) and antimicrobial susceptibility testing was performed by Disk Diffusion Test Methodology as described in the European Committee on
Antimicrobial Susceptibility Testing (EUCAST, Version 1.0, December 18, 2009) or by The Vitek 2 automated system by use of cards AST-N209 and AST-N122 (bioMérieux, France). Methods were performed according to guidelines of DCM in agreement with direction from manufacturers and as described elsewhere [8-11]. The ATCC 25922 E. coli was used for quality control and susceptibility interpreted as recommended by EUCAST (www.eucast.org/clinical_ breakpoints/). Resistance to cefpodoxime was used as an indicator for ESBL-production. Isolates resistant to cefpodoxime therefore had potential ESBL phenotypes identified by a double-disk diffusion method; here performed as combined-disk diffusion using a AmpC + ESBL detection set (MAST ${ }^{\circ}$, Merseyside, UK) as previously described [10].

\section{Molecular characterization ESBL-genotyping}

ESBL-producing E. coli, phenotypically recognized by the double-disk diffusion MAST ${ }^{\bullet}$ test, were screened for the presence of blaCTX-M genes by multiplex PCR assay, detecting alleles encoding the five CTX-M groups $1,2,8,9$ and 25 as previously described [7, 12]. Positive samples were re-amplified and products were sequenced to identify the exact genotypes of CTX-M $[7,12]$.

\section{MLVA and MLST}

All included isolates were typed using a-MLVA, with PCR amplification of six variable number of tandem repeats (VNTR) loci, as described by Nielsen et al. [7]. In short, PCR were done as singleplex PCR with unlabelled primers. Subsequent size determination were done using an automated capillary electrophoresis system (QIAxcel, Qiagen) and a high-resolution cartridge [7]. Each of the VNTR loci was manually binned depending on size and assigned a number. As result each isolate were given a six-digit aMLVA code. For isolates without a measured band size, for a VNTR locus, PCR amplification was performed twice for the given locus. The identified codes were primarily translated to STs by an in-house library, but to further confirm our findings, at least one isolate from each identified aMLVA code were verified by MLST, with the exception of one a-MLVA code. In groups with several isolates having identical a-MLVA codes, we included isolates with extreme band sizes within bins, to effectively cohere clusters. In the ESBL population we furthermore included isolates, belonging to ST131, for O-serogrouping, performed by GlycoVaxyn. In all, MLST was performed on 31 isolates from the ESBL population, on 45 isolates from the resistant population and on 52 isolates from the susceptible population. MLST was done using the Achtmann MLST scheme with PCR and sequencing of seven housekeeping genes followed by assignment of an allelic number from the MLSTdatabase (http://mlst.warwick.ac.uk/mlst/dbs/Ecoli) [7, 13]. 


\section{Results}

\section{Characterization of the $E$. coli populations}

We defined a cluster as three or more isolates identified as the same ST or a-MLVA code. a-MLVA was performed on all isolates and MLST was performed to link a-MLVA codes to an international known nomenclature. Used in this way a-MLVA divides isolates before more laborious and costly typing by MLST. A total of 83 unique a-MLVA codes were identified among the 286 isolates (Additional file 1). In several occasions one ST was subdivided into more than one a-MLVA codes and complex situations existed where the a-MLVA method did not distinguish between two or more STs as has been seen previously with this current a-MLVA method as well as with other MLVA methods [7, 14] (Additional file 1). Results from the MLST are presented in Fig. 1a-c and a maximum likelihood tree showing all ST's found in the three populations is found in Fig. 2. We found 72 different sequence types in the 141 isolates typed by MLST. We discovered ten STs, not previously identified, here labelled "New ST 1-10". We detected a large ST131 cluster with one a-MLVA code primarily belonging to $\mathrm{O} 25$, all of which were resistant to ciprofloxacin. A minor ST131 cluster, with another a-MLVA code belonging to $\mathrm{O} 16$ was likewise detected, but here no isolates showed resistance to ciprofloxacin.

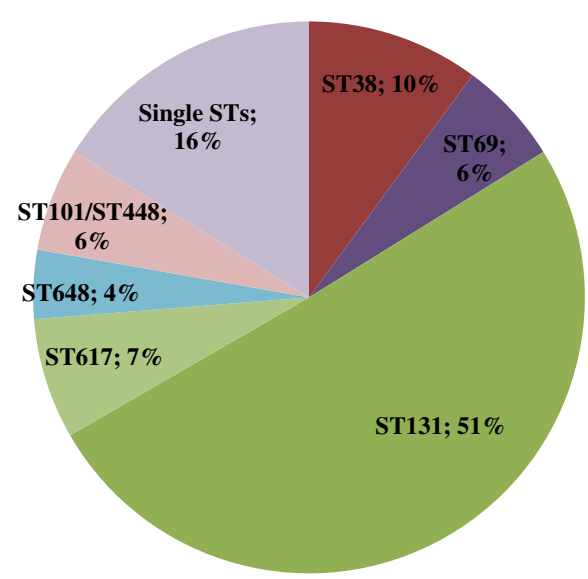

a

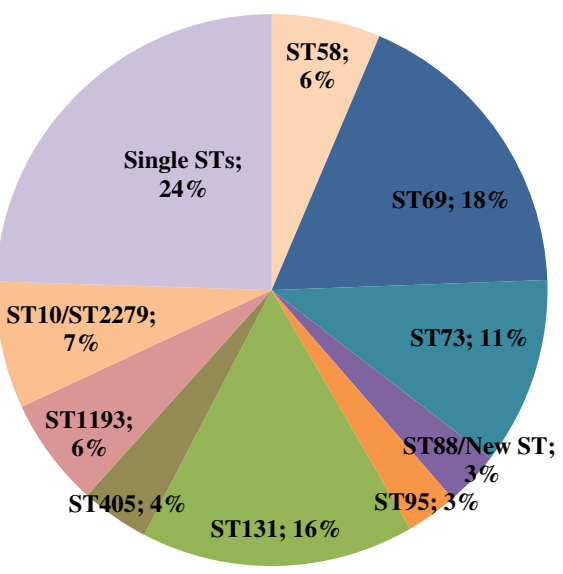

b

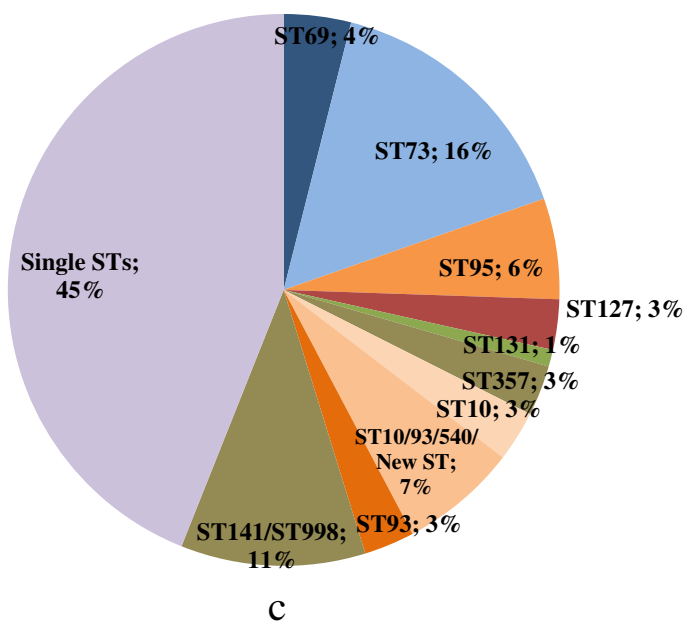

Fig. 1 Distribution of major clusters of sequence types among the three E. coli populations. We show STs making up $2 \%$ or more of isolates-except for ST131 in the susceptible population. Single STs = Percentage of STs found in one isolate only. a ESBL-producing E. coli. STs found but not shown: ST14, ST62, ST88, ST120, ST224, ST315, ST354, ST428, ST636 and ST2852. There was not found a unique a-MLVA code for ST746/ST1598 and ST101/ST448, b Resistant (non-ESBL) E. coli. STs found but not shown: ST14, ST38, ST62, ST80, ST135, ST141, ST362, ST372, ST393, ST457, ST648, ST978, ST1597, New ST (1 and 2) and ST117/ST1177. There was not found a unique a-MLVA code for ST10/ST2279, ST88/New ST 3 and ST117/ST1177. c Susceptible E. coli. STs found but not shown: ST12, ST14, ST38, ST48, ST59, ST62, ST80, ST101, ST127, ST141, ST162, ST405, ST410, ST420, ST501, ST538, ST540, ST582, ST589, ST681, ST714, ST1161, ST1331, ST1444, ST1858, ST3672, ST4235, New ST (4-10) and ST3846. There was not found a unique a-MLVA code for ST10/93/540/New ST and ST141/998 


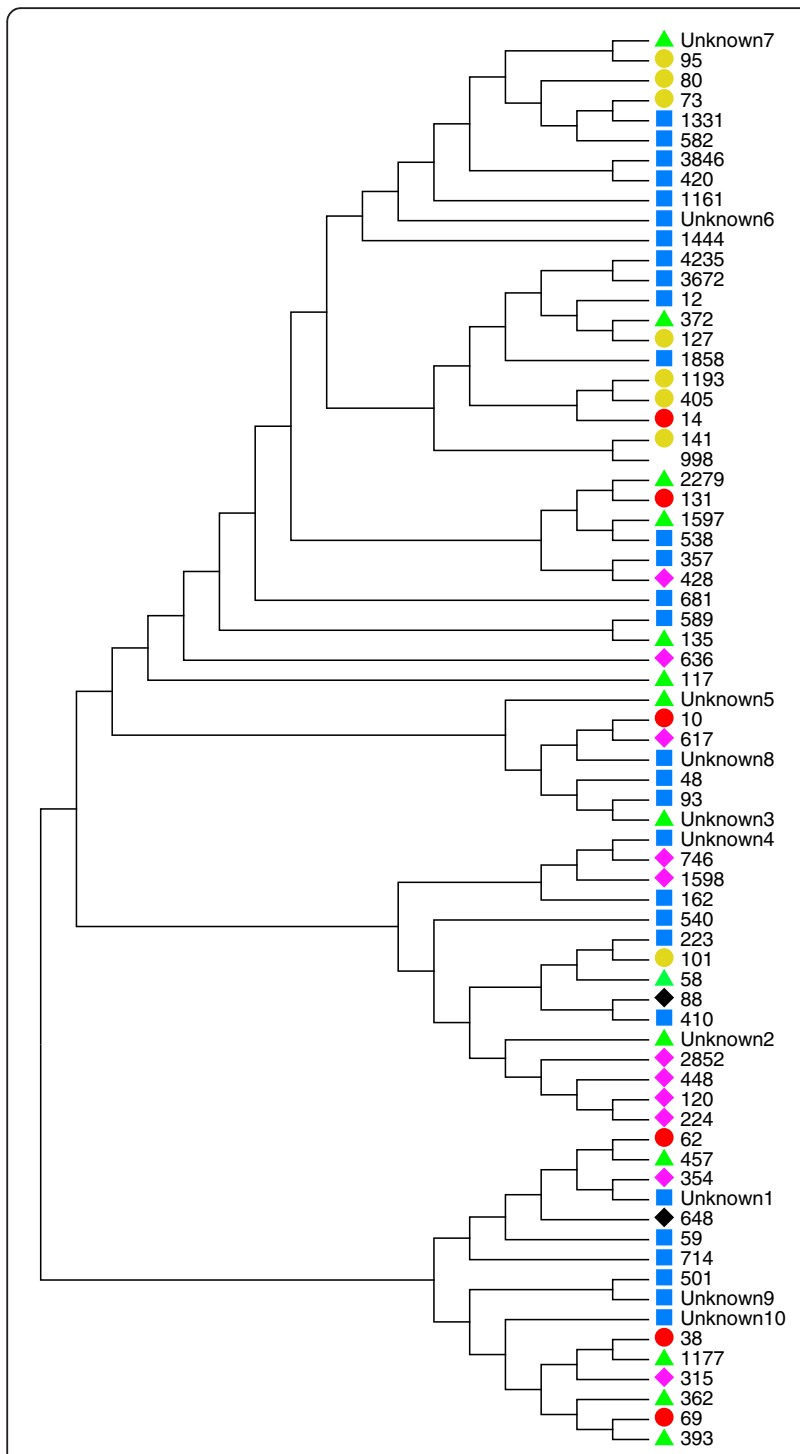

Fig. 2 Maximum likelihood tree showing all ST's found in the three populations. We do not show the number of isolates belonging to each ST. = ST's found in the susceptible population only. $\Delta=$ ST's found in the resistant, non-ESBL population only. $\mathbf{\Delta}=$ ST's found in the ESBL population only. $=$ ST's found in the susceptible and resistant, non-ESBL populations. $=$ ST's found in all three populations. =ST's found in the two resistant populations

\section{Antibiotic resistance patterns}

Results of antibiotic susceptibility testing are found in Figs. 3 and 4. Generally the ESBL population showed high levels of resistance with $100 \%$ of the isolates showing resistance towards three or more of the tested antibiotics. In the resistant population $66 \%$ of the isolates were resistant to $\geq 3$ of the antibiotics. All isolates were susceptible to meropenem and most to mecillinam (97$99 \%$ ), fosfomycin (96-97\%), nitrofurantoin (93-96\%), and piperacillin/tazobactam (92-95\%). Figure 3 indicates

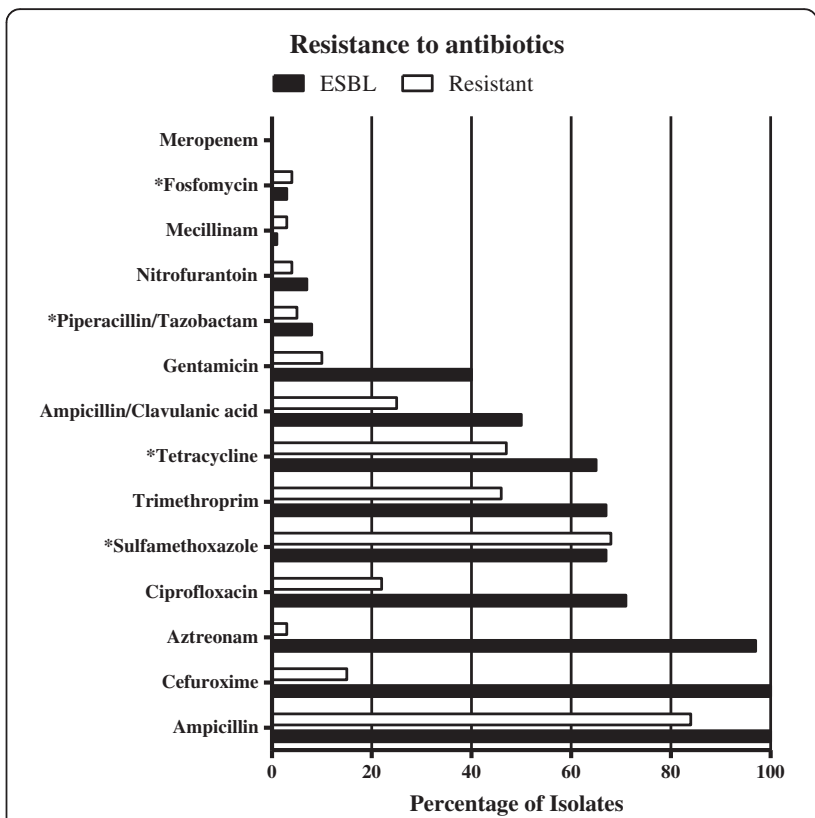

Fig. 3 Resistance patterns for the resistant populations

that resistant (non-ESBL) ST131 have a broader spectrum of resistance than resistant (non-ESBL) ST73 and ST69.

\section{ESBL-genotyping}

We found that $98 \%$ of the ESBL-producing E. coli isolates were positive for the presence of a bla $a_{\mathrm{CTX}-\mathrm{M}}$.

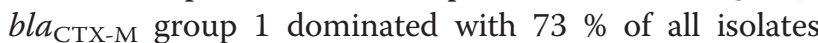
carrying this type and $54 \%$ produced CTX-M-15. A total of $24 \%$ of isolates belonged to CTX-M group 9.

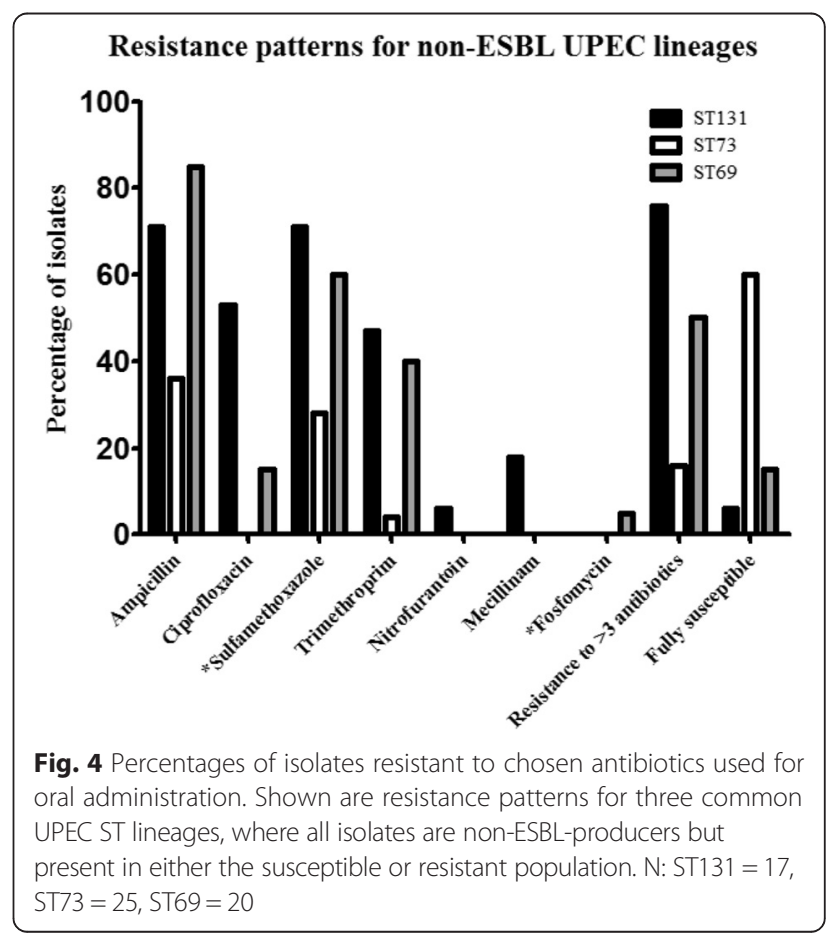




\section{Discussion}

This study was carried out to describe the diversity among susceptible, resistant (non-ESBL) and ESBLproducing E. coli. MLST was performed to link a-MLVA codes to an international known nomenclature. Overall we found ST131, ST73 and ST69 to be the dominating lineages among all isolates, in accordance with previous studies [15]. The resistant (non-ESBL) ST131 showed a broader spectrum of resistance than the other prevalent STs in this population (ST73 and ST69) (Fig. 3), which has also been previously described by Horner et al. [15]. We found that ESBL-producing $E$. coli, resistant $E$. coli and susceptible $E$. coli in turn were dominated by different lineages based on a-MLVA codes and STs (Fig. 1). In general, the susceptible $E$. coli population was a much more diverse group of isolates with more STs and smaller clusters (Fig. 1 and Additional file 1). The STclusters in this population were subdivided by a-MLVA, emphasizing a higher level of diversity. The resistant $E$. coli and ESBL-producing E. coli were found in larger clusters. Especially the ESBL-population was a more homogenous population with fewest a-MLVA codes and STs (Fig. 1). We found that ST131 completely dominated this ESBL-producing E. coli population, as previously seen in the Copenhagen area [7, 16]. Only a single ST131 isolate was found among the susceptible isolates and 14 detected in the resistant population (Fig. 1). Corresponding well with preceding reports, we found that most ST131 isolates belong to serogroup O25 and a minor part being identified as O16 [16, 17]. It should be noted, that the ESBL-population is likely a more selected group, allowing us to detect less successful STs with limited impact as UPEC, which will influence the established representation of diversity in this group. However, we speculate if resistance in different $E$. coli populations are somewhat defined by intrinsic differences in distinct E. coli lineages, making a limited number of UPEC STlineages capable of obtaining and spreading bla $a_{\mathrm{CTX}-\mathrm{M}}$. Some lineages successfully acquire and maintain different types of mobile resistance genes like ST69 and especially ST131, while other E. coli lineages remain fairly susceptible and rarely take up plasmids, as seen with ST73 and ST95 [3, 4]. ST131 could be one of the UPEC lineages, among these resistant and ESBL-producing isolates, with the highest ability to colonize the human gut, creating a high prevalence in these populations. Nevertheless, production of ESBLs are found among specific UPEC present, to some extent, in all populations. Thus, antibiotic selection creates a less varied population structure of related isolates while antibiotic free environments allows for competition and diverse non-related population structure [18]. The UPEC we found in our three populations have been found in other population-studies of $E$. coli isolates causing bacteraemia, strongly indicating that E. coli isolates capable of one invasive disease can cause severe invasive infections, independent of antibiotic susceptibility [15]. Finally, our results are in line with the reports, using more than one method of characterization, identifying susceptible ST-lineages as more heterogeneous populations $[2,6,17,19]$.

One of the major limitations of this study is the incomplete separation of all STs. However, this also provides high discriminatory power in the ability to separate closely related ST-lineages and thereby describe E. coli populations in detail. Yet, the missing identification of STs are unlikely to cause bias in our overall conclusion as the prevalence and identification of the major lineages in the three populations are unique. The aMLVA typing method was successful in the characterization and sub-division of some large cluster of ST-lineages. The study is additionally limited by the relatively small number of isolates characterized and the limited time period of collection, which does not allow us to identify any fluctuation in dominant lineages of non-ESBL populations.

\section{Conclusions}

In conclusion; this study was carried out to describe the diversity among susceptible, resistant (non-ESBL) and ESBL-producing E. coli. Overall we found ST131, ST73 and ST69 to be the dominating lineages among all isolates, in accordance with previous studies [15]. ST131 was able to effectively dominate the ESBL-producing UPEC and seems to be a specialized UPEC adapted as an efficient resistant and ESBL-producing lineage, sustainable in the community once present. The findings we present here strongly suggest that the observed dissemination of ESBL-producing $E$. coli are due to the spread of certain UPEC lineages already present in the general UPEC population [3]. Such lineages seems able to dominate the ESBL-population and it is likely that the spread of resistance occurs due to selection of previously specialized UPEC with limited fitness loss due to ESBLproduction [4].

\section{Availability of supporting data}

We have not deposited additional or supporting data online, but we present the data on which our findings are based in the Additional file 1 for the present paper.

\section{Additional file}

Additional file 1: The additional data contains the characterization of the E.coli populations. It shows the distribution of a-MLVA codes and Sequence Types in details in three tables: Additional file 1: S1 to S3. Here we show the number of isolates found in each cluster of STs and a-MLVA codes, respectively. Furthermore, we present each ST and the 
corresponding a-MLVA code (or a-MLVA codes in case more than one a-MLVA code was identified for a specific ST). In addition, we show cases where more than one ST was identified by the same a-MLVA. Finally, for the ESBL-population, we show the ESBL genotype identified within each a-MLVA code. (PDF $439101 \mathrm{~kb}$ )

\section{Abbreviations}

a-MLVA: abbreviated MLVA; ESBL: extended-spectrum-beta-lactamase; EXPEC: extraintestinal pathogenic Escherichia coli; MALDI-TOF: matrix-assisted laser desorption ionization-time of flight mass spectrometry; MLST: multi locus sequence typing; MLVA: multi locus variable number of tandem repeat analysis; PCR: polymerase chain reaction; ST: sequence type; UPEC: uropathogenic E.coli; UTI: urinary tract infection; VNTR: variable number of tandem repeats.

\section{Competing interest}

The authors declare that they have no competing interests.

\section{Authors' contributions}

All authors contributed to the study design, interpretation of the data, intellectual discussion and/or revision of the manuscript. $\mathrm{FBH}, \mathrm{JBH}, \mathrm{KS}$ and $\mathrm{PL}$ planned and organized the lab work. FBH and JBN performed the work in the laboratory. All data were analyzed by FBH, JBN, KS and PL. FBH, JBN, KS, JDK, ALO and NFM participated in planning of statistical analyses and were responsible for the original study idea and study design. FBH wrote the initial draft of the manuscript. All authors have read and approved the final version of the manuscript before submission.

\section{Acknowledgements}

This investigation was performed with financial support from PAR7, an EU-FP7-Health-2009-Single-Stage project, DanCARD (Danish National Strategic Research Foundation) (No. 09-067075), Roskilde University and the SSAC Foundation (No. SLS-327421 and SLS-251761). Furthermore, work has been performed as cooperation between Hvidovre University Hospital and University of Copenhagen. This work was, in parts, presented at ECCMID in Barcelona 2014

\section{Author details}

${ }^{1}$ Department of Clinical Microbiology, Hvidovre University Hospital, Copenhagen, Denmark. ${ }^{2}$ Department of Biology, University of Copenhagen, Copenhagen, Denmark. ${ }^{3}$ Depatment of Clinical Microbiology, Rigshospitalet, Copenhagen, Denmark.

\section{Received: 24 July 2015 Accepted: 29 March 2016}

\section{Published online: 11 April 2016}

\section{References}

1. Croxall G, Hale J, Weston V, Manning G, Cheetham P, Achtman M, Mcnally A. Molecular epidemiology of extraintestinal pathogenic Escherichia coli isolates from a regional cohort of elderly patients highlights the prevalence of ST131 strains with increased antimicrobial resistance in both community and hospital care settings. 2011(August):2501-2508.

2. Banerjee R, Johnston B, Lohse C, Chattopadhyay S, Tchesnokova V, Sokurenko EV, Johnson JR. Antimicrob Agents Chemother. 2013;57(12):5912. DOI:10.1128/AAC.01065-13.

3. Adams-Sapper S, Diep BA, Perdreau-Remington F, Riley LW. Clonal composition and community clustering of drug-susceptible and-resistant Escherichia coli isolates from bloodstream infections. Antimicrob Agents Chemother. 2013;57:490-7.

4. Bengtsson S, Naseer U, Sundsfjord A, Kahlmeter G, Sundqvist M. Sequence types and plasmid carriage of uropathogenic Escherichia coli devoid of phenotypically detectable resistance. J Antimicrob Chemother. 2012;67:69-73.

5. Nicolas-Chanoine M-H, Blanco J, Leflon-Guibout V, Demarty R, Alonso MP, Caniça MM, Park Y-J, Lavigne J-P, Pitout J, Johnson JR. Intercontinental emergence of Escherichia coli clone O25:H4-ST131 producing CTX-M-15. J Antimicrob Chemother. 2008;61:273-81.

6. Blanco J, Mora A, Mamani R, López C, Blanco M, Dahbi G, Herrera A, Blanco JE, Alonso MP, García-Garrote F, Chaves F, Orellana MÁ, Martínez-Martínez L, Calvo J, Prats G, Larrosa MN, González-López JJ, López-Cerero L, Rodríguez-Baño J, Pascual A. National survey of Escherichia coli causing extraintestinal infections reveals the spread of drug-resistant clonal groups O25b:H4-B2-ST131, O15:H1-D-ST393 and CGA-D-ST69 with high virulence gene content in Spain. J Antimicrob Chemother. 2011; 66:2011-21.

7. Nielsen JB, Albayati A, Jørgensen RL, Hansen KH, Lundgren B, Schønning K. An abbreviated MLVA identifies Escherichia coli ST131 as the major extended-spectrum $\beta$-lactamase-producing lineage in the Copenhagen area. Eur J Clin Microbiol Infect Dis. 2013;32:431-6.

8. Livermore DM, Struelens M, Amorim J, Baquero F, Bille J, Canton R, Henning S, Gatermann S, Marchese a, Mittermayer H, Nonhoff C, Oakton K, Praplan F, Ramos H, Schito GC, Van Eldere J, Verhaegen J, Verhoef J, Visser MR. Multicentre evaluation of the VITEK 2 advanced expert system for interpretive reading of antimicrobial resistance tests. J Antimicrob Chemother. 2002;49:289-300.

9. Nyberg SD, Meurman O, Jalava J, Rantakokko-Jalava K. Evaluation of detection of extended-spectrum beta-lactamases among Escherichia coli and klebsiella spp. Isolates by VITEK 2 AST-N029 compared to the agar dilution and disk diffusion methods. Scand J Infect Dis. 2008;40:355-62.

10. Hansen F, Olsen SS, Heltberg O, Justesen US, Fuglsang-Damgaard D, Knudsen JD, Hammerum AM. Characterization of third-generation cephalosporin-resistant Escherichia coli from bloodstream infections in Denmark. Microb Drug Resist. 2014;00:1-9.

11. Haigh J, Degun A, Eydmann M, Millar M, Wilks M. Improved performance of bacterium and yeast identification by a commercial matrix-assisted laser desorption ionization-time of flight mass spectrometry system in the clinical microbiology laboratory. J Clin Microbiol. 2011;49:3441.

12. Jørgensen RL, Nielsen JB, Friis-Møller A, Fjeldsøe-Nielsen H, Schønning K. Prevalence and molecular characterization of clinical isolates of Escherichia coli expressing an AmpC phenotype. J Antimicrob Chemother. 2010;65:460-4.

13. Wirth T, Falush D, Lan R, Colles F, Mensa P, Wieler LH, Karch H, Reeves PR, Maiden MCJ, Ochman H, Achtman M. Sex and virulence in Escherichia coli: an evolutionary perspective. Mol Microbiol. 2006;60:1136-51.

14. Naseer U, Olsson-Liljequist BE, Woodford N, Dhanji H, Cantón R, Sundsfjord A, Lindstedt B-A. Multi-locus variable number of tandem repeat analysis for rapid and accurate typing of virulent multidrug resistant Escherichia coli clones. PLoS One. 2012;7:e41232.

15. Horner C, Fawley W, Morris K, Parnell P, Denton M, Wilcox M. Escherichia coli bacteraemia: 2 years of prospective regional surveillance (2010-12). J Antimicrob Chemother. 2014;69:91-100.

16. Olesen B, Hansen DS, Nilsson F, Frimodt-Møller J, Leihof RF, Struve C, Scheutz F, Johnston B, Krogfelt K a, Johnson JR. Prevalence and characteristics of the epidemic multiresistant Escherichia coli ST131 clonal group among extended-spectrum beta-lactamase-producing E. Coli isolates in Copenhagen, Denmark. J Clin Microbiol. 2013;51:1779-85.

17. Peirano G, van der Bij AK, Gregson DB, Pitout JDD. Molecular epidemiology over an 11-year period (2000 to 2010) of extended-spectrum $\beta$-lactamaseproducing Escherichia coli causing bacteremia in a centralized Canadian region. J Clin Microbiol. 2000;2012:294-9.

18. Maiden MCJ. Multilocus sequence typing of bacteria. Annu Rev Microbiol. 2006;60:561-88

19. Johnson JR, Tchesnokova V, Johnston B, Clabots C, Roberts PL, Billig M, Riddell K, Rogers P, Qin X, Butler-Wu S, Price LB, Aziz M, Nicolas-Chanoine M-H, Debroy C, Robicsek A, Hansen G, Urban C, Platell J, Trott DJ, Zhanel G, Weissman SJ, Cookson BT, Fang FC, Limaye AP, Scholes D, Chattopadhyay S, Hooper DC, Sokurenko E V. Abrupt emergence of a single dominant multidrug-resistant strain of Escherichia coli. J Infect Dis. 2013;207:919-28. 